\title{
Amphiphilic beads as depots for sustained drug release integrated into fibrillar scaffolds
}

\author{
Akhilesh K. Gaharwar a,b,c,j,k , Silvia M. Mihaila b,d,e,f ${ }^{\text {, }}$ Ashish A. Kulkarni ${ }^{\text {b,d }}$, Alpesh Patel ${ }^{\text {b,d }}$, Andrea Di Luca ${ }^{\mathrm{g}}$, \\ Rui L. Reis ${ }^{\mathrm{e}, \mathrm{f}}$, Manuela E. Gomes ${ }^{\mathrm{e}, \mathrm{f}}$, Clemens van Blitterswijk ${ }^{\mathrm{g}}$, Lorenzo Moroni ${ }^{\mathrm{g}, *}$, Ali Khademhosseini ${ }^{\mathrm{a}, \mathrm{b}, \mathrm{d}, \mathrm{h}, \mathrm{i}, * * *}$ \\ ${ }^{a}$ Wyss Institute for Biologically Inspired Engineering, Harvard University, Boston 02115, USA \\ b Biomaterials Innovation Research Center, Department of Medicine, Brigham and Women's Hospital, Harvard Medical School, Cambridge 02139, USA \\ ${ }^{c}$ David H. Koch Institute for Integrative Cancer Research, Massachusetts Institute of Technology, Cambridge 02139, USA \\ d Harvard-MIT Division of Health Sciences and Technology, Massachusetts Institute of Technology, Cambridge 02139, USA \\ e 3B's Research Group, Biomaterials, Biodegradables and Biomimetics, Dept. of Polymer Engineering, University of Minho, AvePark, Taipas, 4806-909 Guimarães, Portugal \\ ${ }^{\mathrm{f}}$ ICVS/3B's-PT Government Associate Laboratory, Braga, Guimarães, Portugal \\ g Tissue Regeneration Department, MIRA Institute for Biomedical Technology and Technical Medicine, University of Twente, Enschede, Netherlands \\ ${ }^{\mathrm{h}}$ Department of Maxillofacial Biomedical Engineering and Institute of Oral Biology, School of Dentistry, Kyung Hee University, Seoul 130-701, Republic of Korea \\ ${ }^{i}$ Department of Physics, King Abdulaziz University, Jeddah 21569, Saudi Arabia \\ ${ }^{\mathrm{j}}$ Department of Biomedical Engineering, Texas A\&'M University, College Station 77843, USA \\ ${ }^{\mathrm{k}}$ Department of Materials Science \& Engineering, Texas AEM University, College Station 77843, USA
}

\section{A R T I C L E I N F O}

\section{Article history:}

Received 10 April 2014

Accepted 21 April 2014

Available online 29 April 2014

\section{Keywords:}

Electrospinning

Drug release

Human mesenchymal stem cells

Fibrous scaffolds

Amphiphilic polymer

\begin{abstract}
A B S T R A C T
Native extracellular matrix (ECM) is a complex fibrous structure loaded with bioactive cues that affects the surrounding cells. A promising strategy to mimicking native tissue architecture for tissue engineering applications is to engineer fibrous scaffolds using electrospinning. By loading appropriate bioactive cues within these fibrous scaffolds, various cellular functions such as cell adhesion, proliferation and differentiation can be regulated. Here, we report on the encapsulation and sustained release of a model hydrophobic drug (dexamethasone (Dex)) within beaded fibrillar scaffold of poly(ethylene oxide terephthalate)-poly(butylene terephthalate) (PEOT/PBT), a polyether-ester multiblock copolymer to direct differentiation of human mesenchymal stem cells (hMSCs). The amphiphilic beads act as depots for sustained drug release that is integrated into the fibrillar scaffolds. The entrapment of Dex within the beaded structure results in sustained release of the drug over the period of 28 days. This is mainly attributed to the diffusion driven release of Dex from the amphiphilic electrospun scaffolds. In vitro results indicate that hMSCs cultured on Dex containing beaded fibrillar scaffolds exhibit an increase in osteogenic differentiation potential, as evidenced by increased alkaline phosphatase (ALP) activity, compared to the direct infusion of Dex in the culture medium. The formation of a mineralized matrix is also significantly enhanced due to the controlled Dex release from the fibrous scaffolds. This approach can be used to engineer scaffolds with appropriate chemical cues to direct tissue regeneration.
\end{abstract}

(c) 2014 Elsevier B.V. All rights reserved.

\section{Introduction}

Native extracellular matrix (ECM) is a complex fibrous structure that provides physical, chemical, and mechanical cues to direct cellular processes [1-5]. A promising strategy to mimic the native tissue architecture is to engineer fibrous scaffolds using electrospinning (ESP) technique [6]. By incorporating appropriate topographical or therapeutic/ bioactive cues within the fibrous scaffolds, various cellular processes

\footnotetext{
* Corresponding author.

** Correspondence to: A. Khademhosseini, Center for Biomedical Engineering, Department of Medicine, Brigham and Women's Hospital, Harvard Medical School, Cambridge 02139, USA.

E-mail addresses: l.moroni@utwente.nl (L. Moroni), alik@rics.bwh.harvard.edu (A. Khademhosseini)
}

can be controlled to facilitate the formation of musculoskeletal tissues [7-9]. For example, these fibrous scaffolds could find applications as bone fillers, in non-load bearing defects such as skull defects, or as bone membranes such as in the case of periosteum regeneration [7-9]. Electrospun scaffolds composed of hydroxyapatite/chitosan have shown to promote new bone regeneration in vivo by activating integrin and bone morphogenetic protein (BMP)/Smad signaling pathway [10]. Fibrous membranes composed of gelatin/polycaprolactone have shown to promote in vitro and in vivo cartilage tissue regeneration [11]. In a similar study, fibrous scaffolds made from poly(L-lactide-co- $\varepsilon$ caprolactone)/collagen ( $\mathrm{P}(\mathrm{LLA}-\mathrm{CL}) / \mathrm{Col})$ stimulated the differentiation of tendon-derived stem cells when subjected to mechanical stimulation [12].

Even when load bearing applications are considered, electrospun scaffolds can be used in combination with, for example, rapid prototyped 
scaffolds with mechanical properties matching those of bone [13]. In this respect, the electrospun scaffolds can be useful to deliver biological factors that can augment, trigger, sustain and enhance the regenerative process. Silk fibroin based electrospun scaffolds loaded with bone morphogenetic protein 2 (BMP-2) have shown to promote mineralized matrix formation in vitro due to release of BMP-2 [14]. The surface of electrospun fibers can be functionalized to load appropriate bioactive moieties to control cell fate [15-17]. To obtain a 3D porous network, a range of techniques, such as the use of porogenic materials or watersoluble agents are proposed [18]. After subjecting the electrospun scaffolds loaded with these agents to water, the desired porosity can be achieved [18]. Another technique to enhance the porosity of electrospun scaffolds includes laser ablation [19]. This technique allows incorporation of micromachined pores with predetermined dimension and location to improve cellular infiltration.

A range of hydrophobic or hydrophilic therapeutic agents can be incorporated within electrospun fibers by blending them with the polymer solution prior to electrospinning [20-23]. The entrapped therapeutic/bioactive molecules can be released in vitro and in vivo as part of the volumetric or surface matrix or as a soluble factor in a sustained and controlled manner to control cellular behaviors. For example, bioactive agents such as BMPs [24,25], dexamethasone [26,27], hydroxyapatite [28,29], calcium phosphate [30] and silicate nanoparticles [31-33] are incorporated within polymeric scaffolds to induce osteogenic differentiation of stem cells. The release rate of these bioactive moieties can be modified by altering the fiber morphology, degradation rate, hydrophilicity of polymer and drug loading $[9,23,34,35]$.

Dexamethasone (Dex) is a synthetic member of the glucocorticoid class of steroid drugs and is used in the treatment of severe inflammatory diseases [36]. Dex has a concentration-dependent stimulatory effect on the differentiation of human mesenchymal stem cells (hMSCs) [37, 38]. For example, hMSCs treated with Dex show increased levels of alkaline phosphatase (ALP) activity, which is an early marker for osteogenic differentiation [39]. Furthermore, Dex is also known to enhance matrix mineralization of hMSCs in combination with $\beta$-glycerolphosphate and ascorbic acid [40]. Although the exact mode of action by which Dex functions is unidentified, it is known that it enters the cell where it binds to specific regulatory proteins, thereby, activating the transcription of osteoblast-specific genes [26]. Although Dex is known to have a prolonged effect on ALP expression and matrix mineralization even after only a few days of exposure [41], continuous treatment of hMSCs with Dex results in the most efficient induction of differentiation and subsequent matrix mineralization [42].

To control the release of Dex, various strategies such as encapsulation (or entrapment/attachment) within poly(lactic-co-glycolic acid (PLGA) microspheres [43], carbon nanotubes [44,45], poly(amidoamine) (PAMAM) dendrimer nanoparticles [46] and hyperbranched polyester hydrogels [47] have been reported. However, limited research has been focused on controlled delivery of Dex from electrospun scaffolds [48-51]. Martins et al. showed an increase in ALP expression and matrix mineralization of hMSCs on electrospun polycaprolactone (PCL)/Dex meshes in a basal medium containing $\beta$-glycerophosphate compared to the unloaded meshes in an osteogenic medium [48,51]. This study demonstrated that controlled release of Dex is an improvement over normal dexamethasone-in-medium culture conditions [48,51]. However, due to the crystalline nature of PCL, the sustained release of Dex over long periods of time was not observed and a plateau phase was reached within 4-5 days. This might be due to the formation of Dex aggregates within the PCL scaffolds over time that results in limited release of the entrapped drug. Moreover, the amount of Dex required to induce osteogenic differentiation was not compatible with the standard concentration used in the established osteogenic differentiation protocols. At the same time, it was shown that high concentrations of Dex could impair cell proliferation and trigger the upregulation of adipogenesis in parallel with osteogenesis (in vitro) [52]. Therefore, it is important to tune Dex release rate from any carrier-device according to the strict requirements to obtain an efficient osteogenesis, followed by a robust mineralization.

Recently, Nguyen et al. fabricated Dex loaded poly(L-lactic acid) (PLLA) nanofibrous scaffolds [49]. They also observed that the release of Dex from these electrospun fibers induces differentiation of hMSCs over a period of 3 weeks. In a similar approach, Vacanti et al. entrapped Dex within electrospun fibers of PLLA and PCL [50]. Entrapped Dex was released from PCL scaffolds within $24 \mathrm{~h}$, whereas from PLLA a sustained delivery for longer time frame was observed. They also demonstrated that the localized in vivo delivery of Dex evoked a less severe inflammatory response when compared with only PCL or PLLA fibers.

Although, encapsulation of Dex in hydrophobic polymers such as PCL and PLLA is described, to our knowledge the release of Dex from amphiphilic polymers has not been reported. Amphiphilic block polymers with tailored physical and chemical properties have shown a controlled release profile and linear degradation characteristics that can be used for a range of tissue engineering applications [34,53,54]. We hypothesize that entrapping Dex within bead-like depots in an amphiphilic fibrillar scaffold will result in a sustained release profile over longer duration. Among amphiphilic copolymers, random block copolymers of poly(ethylene oxide) terephthalate and poly(butylene terephthalate) (PEOT/PBT) have been extensively investigated due to their bioactive characteristics $[34,55,56]$. By varying the molecular weight and polymer composition, a wide range of PEOT/PBT copolymer with the desired mechanical strength, hydration properties, degradation profiles and biological characteristics can be obtained [57]. The PEOT/PBT copolymers are biodegradable and have been proposed for osteochondral tissue engineering [58-60]. 3D scaffolds from PEOT/PBT were fabricated by using 3D fiber deposition (3DF) and electrospinning (ESP) and showed to enhance cartilage tissue formation [61]. Due to the amphiphilic nature of PEOT/PBT, it is predicted that hydrophobic drugs (such as Dex) can be entrapped within the polymeric structure and sustained release profiles from the fibrillar structure can be obtained. It is envisioned that such scaffold design can be used for a range of musculoskeletal tissues engineering applications that require control release of hydrophobic drugs to promote tissue regeneration.

In this study, electrospun scaffolds of PEOT/PBT containing different loadings of Dex were prepared. The surface morphologies of these fibers were examined by scanning electron microscopy (SEM). The entrapment of Dex and in vitro release kinetics were investigated using spectroscopic and chromatography techniques. The ability of Dex loaded fibers to control hMSC adhesion, proliferation and differentiation was also investigated. We hypothesize that hMSCs cultured on Dex releasing scaffolds will show enhanced osteogenic differentiation compared to the direct infusion of Dex in medium. The proposed approach for directing cellular function by the sustained release of a hydrophobic drug from amphiphilic fibrous scaffolds can be used to engineer a range of biomimetic scaffolds for controlled drug delivery and regenerative medicine applications.

\section{Experimental}

\subsection{Fabrication of PEOT/PBT electrospun scaffolds}

PEOT/PBT was obtained from PolyVation B.V. (Groningen, The Netherlands). The composition used in this study was 1000PEOT70PBT30 where, 1000 is the molecular weight in $\mathrm{g} / \mathrm{mol}$ of the starting poly(ethylene glycol) (PEG) blocks used in the copolymerization, while 70 and 30 are the weight ratios of the PEOT and PBT blocks, respectively. PEOT is a hydrophilic polymer that imparts elastomeric properties, whereas PBT is a thermoplastic crystalline polymer and imparts stiffness to the copolymeric network. The fibrous scaffolds were fabricated by ESP. First, PEOT/ PBT $(20 \% w / v)$ was dissolved in a 9:1 ratio of anhydrous chloroform and ethanol. ESP was carried out at $12.5 \mathrm{kV}$ (Glassman High Voltage, INC) using a $21 \mathrm{G}$ blunt needle and a flow rate of $2 \mathrm{~mL} / \mathrm{h}$. The collector was a circular plate (diameter $6.5 \mathrm{~cm}$ ) made of aluminum and maintained at 
a constant distance of $18 \mathrm{~cm}$ from the needle. The electrospun scaffolds were dried overnight in vacuum to remove the residual solvent. For the preparation of the Dex loaded PEOT/PBT scaffolds, the drug was dissolved in ethanol $(10 \times$ the desired final concentration) and then dissolved in 9 parts of chloroform. PEOT/PBT solutions containing $0.5,1$ and $2 \%$ of Dex $(w t / w t)$ were prepared. ESP was carried out as described above.

\subsection{Scanning electron microscopy}

The size and morphologies of the electrospun fibers were evaluated using a scanning electron microscope (JSM 5600LV, JEOL USA Inc., MA). The fibers were allowed to dry in a desiccator for $24 \mathrm{~h}$ before imaging. The scaffolds were coated with $\mathrm{Au} / \mathrm{Pd}$ for 2 min using a Hummer 6.2 sputter coater (Ladd Research, Williston, VT). All images were captured using $5 \mathrm{kV}$ acceleration voltage and a working distance of 5-10 mm. Image software (National Institute of Health) was used to determine the size of the fibers from the SEM micrographs. The diameter of at least 50 fibers was measured from one image to determine the average fiber diameter. The bead was excluded while determining the fiber diameter of the electrospun fibers. The bead density was calculated manually by counting the number of beads in an image and then dividing it by the total area.

\subsection{Chemical characterization}

Fourier transform infrared (FTIR) spectra of the samples were recorded using an Alpha Bruker spectrometer. The average value of 48 scans at $4 \mathrm{~cm}^{-1}$ resolutions was collected for each sample. Highperformance liquid chromatography (HPLC) was performed to determine the presence of Dex in electrospun scaffolds. The Water 600 system consisted of an automatic sample injector (Waters 717) and a UV absorbance detector (Waters 2487) set at $254 \mathrm{~nm}$. The mobile phase consisted of acetonitrile. The analytical column was $3.9 \mathrm{~mm} \times 300 \mathrm{~mm}$, and pore size $4 \mu \mathrm{m}$ (Millipore Corp, Waters, Milford, $\mathrm{MA}$ ). The flow rate was set at $1 \mathrm{~mL} / \mathrm{min}$. The retention time of Dex was $3.5 \mathrm{~min}$, and the total run time of HPLC analysis was $10 \mathrm{~min}$. The chromatograph was analyzed by Empower Pro software (Waters). For release kinetics studies, drug-loaded electrospun scaffolds $(50 \mathrm{mg}$ in $10 \mathrm{~mL}$ ) were suspended in PBS in a dialysis tube (MWCO $=3500$ Dalton, Spectrum Lab). The dialysis tube was then suspended in $50 \mathrm{~mL}$ PBS with gentle stirring. At predetermined time intervals, $1 \mathrm{~mL}$ portion of PBS was collected for quantification and replaced by equal volume of PBS, and the release of Dex was quantified by HPLC. The thermal properties of scaffolds were investigated using differential scanning calorimetry (DSC). The electrospun scaffold samples (3-5 mg in weight) were sealed in an aluminum pan and were subjected to 2 heating/cooling cycles from $-70{ }^{\circ} \mathrm{C}$ to $100{ }^{\circ} \mathrm{C}$ at a heating rate of $10{ }^{\circ} \mathrm{C} / \mathrm{min}$ under a constant flow of nitrogen at $20 \mathrm{~mL} / \mathrm{min}$. Protein adsorption was determined using micro bicinchoninic acid (micro BCA) protein assay reagent (Pierce BCA, Thermo Scientific). Briefly, electrospun scaffolds were subjected to $10 \%$ fetal bovine serum (FBS) at $37{ }^{\circ} \mathrm{C}$ in PBS for $24 \mathrm{~h}$. Then samples were washed 3 times in PBS to extract any non-specific adsorbed proteins and were treated with a $2 \%$ SDS solution for $6 \mathrm{~h}$ in a shaker ( $50 \mathrm{rpm})$ to extract the adsorbed proteins. The supernatant was collected separately and was analyzed using the manufacturer's protocol.

\subsection{Mechanical properties}

The mechanical properties of electrospun scaffold were evaluated using uniaxial tensile test using an Instron 5943 Materials Testing System Capacity (Norwood, MA, USA) equipped with a $50 \mathrm{~N}$ load cell. The samples were cut into rectangular shapes that were $10 \mathrm{~mm}$ long, $5 \mathrm{~mm}$ wide and approximately $100-150 \mu \mathrm{m}$ thick. The samples were stretched until failure at the crosshead speed of $10 \mathrm{~mm} / \mathrm{min}$. The elastic modulus was calculated from the linear stress-strain region by fitting a straight line between 5 and 20\% strain. The ultimate tensile stress and failure strain were also calculated.

\subsection{In vitro cell culture studies}

Bone marrow-derived hMSCs (PT-2501, Lonza) were cultured in normal growth medium (a-MEM, containing $10 \%$ of heat-inactivated fetal bovine serum (HiFBS, Gibco, USA) and 1\% Pen/Strep (penicillin/ streptomycin, $100 \mathrm{U} / 100 \mu \mathrm{g} / \mathrm{mL}$, Gibco, USA)) at $37{ }^{\circ} \mathrm{C}$, in a humidified atmosphere with $5 \% \mathrm{CO}_{2}$. Prior to cell seeding, the electrospun scaffolds were sterilized using ethanol for $30 \mathrm{~s}$ before cell seeding, followed by thorough washing with PBS. The cells were cultured until 70-75\% confluence and were used before passage 4 for all the experiments. The cells were trypsinized (CC-3232) and seeded on electrospun scaffolds $\left(1 \times 1 \mathrm{~cm}^{2}\right)$ at the density of 20,000 cells/scaffold in normal growth medium. After $24 \mathrm{~h}$, the electrospun PEOT/PBT scaffolds were subjected to growth medium ( - Dex) and osteogenic medium ( + Dex), as negative and positive control, respectively. Whereas, electrospun scaffolds were containing Dex were subjected to medium ( - Dex) to evaluate the effect of released Dex from the electrospun scaffolds on the hMSC differentiation.

Cell proliferation over 21 days of culture was evaluated using Alamar Blue Assay (Invitrogen) following the standard manufacturer protocol. ALP activity was quantified using Alkaline Phosphatase Colorimetric Assay Kit (Abcam, ab83369). The ALP enzyme in cell lysate converts $p$ nitrophenol phosphate (pNPP) (present in kit) to yellow $p$-nitrophenol (pNP) that can be easily detected using colorimetric assay. Briefly, samples and the assay buffer solution ( $5 \mathrm{mM}$ pNPP) were added to a 96-well plate. After $1 \mathrm{~h}$ of incubation, the absorbance was read at $405 \mathrm{~nm}$ using a microplate reader (Epoch microplate reader, Biotek, USA). A standard curve was made from standards $(0-20 \mu \mathrm{M})$ prepared with a pNPP solution. The samples and standard were analyzed and sample concentrations were read from the standard curve $(n=3)$. To detect the expression of ALP, nitro-blue tetrazolium/indolylphosphate (NBT/BCIP) (Thermo Scientific) staining was also performed. Before staining, the cells were washed with PBS, $0.5 \mathrm{~mL}$ of NBT/BCIP was added and then the samples were incubated at $37{ }^{\circ} \mathrm{C}$ in a humidified chamber containing $5 \% \mathrm{CO}_{2}$. After $30 \mathrm{~min}$, the samples were washed with PBS and fixed with $4 \%$ paraformaldehyde for imaging. The optical images of stained scaffold were obtained using Zeiss Axio Observer Z1 1 (AXIO1) equipped with a color camera (Evolve EMCCD $512 \times 51216 \mu \mathrm{m}$ pixels).

\subsection{Statistics}

Experimental data were presented as mean \pm standard deviation ( $n=3$ to 5 ). Statistical differences between the groups were analyzed using one-way ANOVA with Tukey post-hoc analysis for fiber analysis, mechanical testing and drug loading, and two-way ANOVA for ALP analysis. Statistical significance was represented as ${ }^{*} p<0.05,{ }^{* *} p<0.01,{ }^{* * *} p<0.001$.

\section{Results and discussion}

Electrospun fibrous scaffolds are highly porous 3D network structures. The fibrous scaffolds were obtained by ESP of PEOT/PBT copolymer (Fig. 1a). Dex-loaded beaded structures were obtained by mixing PEOT/PBT with different amounts of Dex $(0,0.5,1$ and $2 \% w t / w t$ Dex compared to the polymer) before the ESP process (Fig. 1b). The effect of Dex on the chemical, structural and biological properties of the PEOT/PBT electrospun scaffolds was evaluated.

\subsection{Amphiphilic beads integrated into fibrillar scaffolds}

The morphology and size of electrospun fibers were examined using a scanning electron microscope (Fig. 1c). ESP of PEOT/PBT resulted in 
formation of uniform fiber size $(2.15 \pm 0.7 \mu \mathrm{m})$ with smooth surface morphology. The addition of small amount of Dex (0.5\%) resulted in formation of beaded structures along the fiber. Moreover, a significant decrease in fiber diameter was observed due to addition of Dex. The addition of $0.5,1$ and $2 \%$ Dex significantly decreases the fiber diameter to $0.61 \pm 0.20,0.62 \pm 0.21$ and $0.51 \pm 0.22 \mu \mathrm{m}$ respectively (Fig. $1 \mathrm{~d}$ ). However, with the addition of Dex, the number of beads within the scaffold structure increased (Fig. 1e). This might be attributed to an increase in the conductivity of the polymeric solution due to the addition of Dex. The number and size of beads were quantified using image analysis and results indicated that addition of the Dex resulted in an increase in the number of beads without significantly changing the beads dimension.

The formation of beads due to addition of Dex highlights that these beads can act as drug depots or reservoirs integrated with the electrospun fibrous network. The entrapped drug from these depots might release due to diffusion/degradation of fibers within a controlled fashion and subsequently control cellular behavior and functionality. To determine the location of drug within these fibrous structures, we mixed Texas Red (a fluorescence molecule with similar molecular weight as Dex) with PEOT/PBT solution and fabricated electrospun scaffolds loaded with Texas Red. The microstructure analysis showed that the addition of this fluorescence dye instead of Dex did not result in change in fiber morphology. By observing these beaded structures under fluorescence microscope, the location of dye within the fibrous structure was determined. Fig. 2a showed that the entrapped dye is mainly located within the beaded structure, hence predicting the distribution of Dex analog within the beaded units of the fibers. This indicates that the beaded structures effectively act as reservoirs of the dye or drug molecule. Thus it can be expected that when Dex is mixed with PEOT/
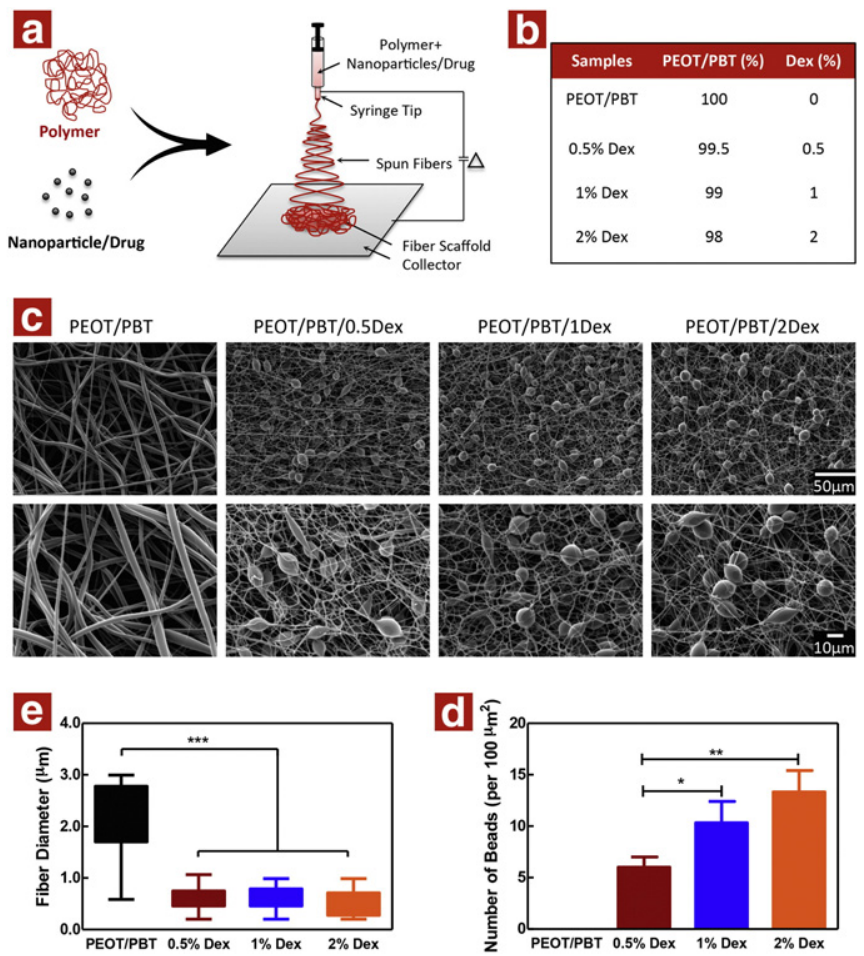

Fig. 1. Formation of electrospun beaded fibers. (a) A schematic showing the formation of electrospun scaffolds by combining PEOT/PBT with Dex. (b) The composition of electrospun fibers is listed. (c) The effect of Dex on fiber diameter and morphology was evaluated using SEM. ESP of PEOT/PBT shows the formation of smooth and uniform fibers. The addition of a small amount of Dex results in fibers with smaller diameters and beaded structures. (d) The box plot representing distribution of fiber diameter is shown; the top and the bottom of the box represent 25 th and 75 th percentile respectively, while whiskers represent min-max value of the fiber diameter $(n=60)$. (e) The addition of Dex results in an increase in the number of beaded structures. The data represents mean \pm standard deviation. (One-way ANOVA with Tukey post-hoc, ${ }^{*} p<0.05,{ }^{* *} p<0.01$, and ${ }^{* * *} p<0.001$ ).
PBT, it might get accumulated within these beaded structure (depots) and these depots were integrated within fibrillar scaffolds.

The effect of the addition of Dex on the thermal and mechanical properties of electrospun fibers was also investigated using differential scanning calorimetry (DSC) and uniaxial tensile test, respectively. The thermal analysis of electrospun fiber indicated no effect on the melting temperature $\left(T_{\mathrm{m}}\right)$ of PEOT/PBT due to addition of Dex (Fig. 2b). This might be due to a very low amount of Dex within the electrospun scaffold compared to the amount of polymer. The addition of Dex resulted in a significant decrease in elastic modulus, ultimate tensile strength and elongation of electrospun fibers (Fig. 2c). This is mainly attributed to the correspondent decrease in the fiber diameter due to the addition of Dex.

\subsection{Sustained release of Dex from beaded fibrillar scaffolds}

Incorporation of Dex within PEOT/PBT scaffolds was evaluated using high-performance liquid chromatography (HPLC) and Fourier transform infrared spectroscopy (FTIR) (Fig. 3). The retention time of PEOT/ PBT was $3.5 \mathrm{~min}$, and for Dex was $4.3 \mathrm{~min}$. The electrospun fibers containing 2\% Dex show peaks for both PEOT/PBT and Dex as shown in Fig. 3a. The loading efficiency of Dex was also investigated by dissolving the electrospun fiber. The results indicated that (Fig. 3b) PEOT/PBT fibers with $0.5,1$ and $2 \%$ Dex have loading of $2.1 \pm 0.8,8.3 \pm 6.1$ and $17.6 \pm 9.3 \mu \mathrm{g}$ of Dex/mg of PEOT/PBT, respectively. The loading efficiency of Dex in PEOT/PBT fibers with $0.5,1$ and $2 \%$ Dex was $42 \pm 16 \%$, $83 \pm 61 \%$, and $88 \pm 46.5 \%$, respectively. The loading efficiency was lower compared to the theoretical value and this might be attributed to the loss of Dex during the ESP process. Similar results were obtained for the other types of drug during ESP [48]. a
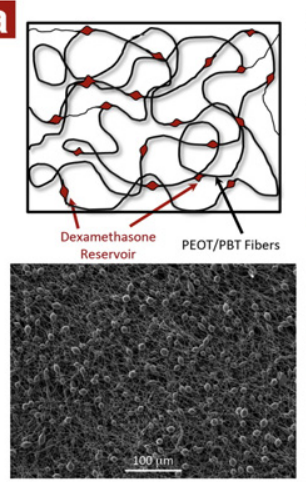

C.

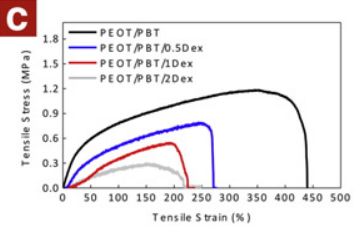

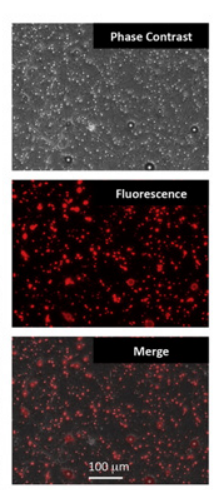

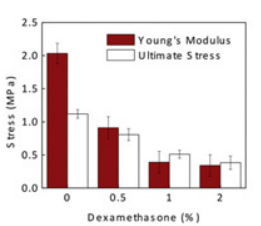

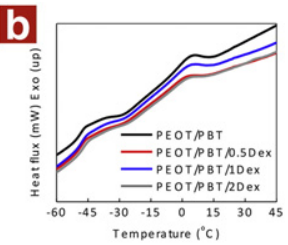
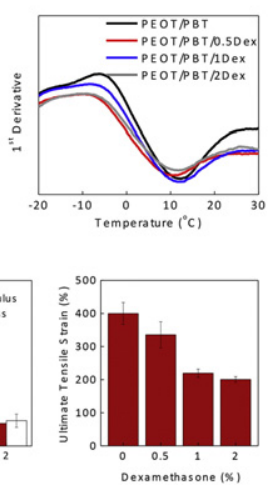

Fig. 2. Beaded structure as drug depot within fibrillar scaffolds. (a) Schematics of the localization of drug within fiber. To determine the localization of Dex within the fibrillar structure, Dex was replaced with a fluorescence dye "Texas Red" due to its similar molecular weight with Dex. Addition of Texas Red (2\% wt/wt) to PEOT/PBT forms a beaded fibrillar structure. Imaging techniques reveal distribution and localization of dye within the beaded structures. Optical microscopy of electrospun fibers loaded with Texas Red displays the beaded structure within fibrillar scaffolds. The fluorescence imaging indicates that the dye is localized within the beaded structure. SEM image of beaded fibers showing uniform distribution of beads within the fibrous scaffolds of PEOT/PBT/2Dex. (b) Effect of Dex on thermal property of PEOT/PBT was investigated using DSC. No significant influence on the $T_{\mathrm{g}}$ of PEOT/PBT was observed due to the addition of Dex. (c) Effect of Dex on the mechanical properties of PEOT/PBT fibers was evaluated using uniaxial tensile test. Formation of the beaded structure due to addition of Dex results in a decrease in mechanical properties such as elastic modulus, tensile strength and ultimate strain. This might be attributed to a decrease in the fiber diameter and formation of the beaded structure. The number of beads present within a scaffold directly depends on the Dex concentration. The data represents mean \pm standard deviation $(n=5)$. 
The presence of Dex within the electrospun fibers was further verified by FTIR and the spectra from Dex only, PEOT/PBT only and Dex loaded PEOT/PBT are shown in Fig. 3c. A characteristic peak at $1660 \mathrm{~cm}^{-1}$ was observed in the loaded electrospun scaffolds indicating the presence of Dex within the scaffolds. This observation is consistent with earlier studies that reported the entrapment of Dex within electrospun fibers [48]. The bioactive agents, such as Dex, need to be delivered over a long period of time, within a controlled and systematic fashion, to direct stem cells into desired lineages and promote the formation of functional tissues [62]. The next generation of intelligent tissue engineered scaffolds should not only facilitate cell adhesion, spreading and proliferation, but should also direct cellular components to synthesize ECM and perform according to the desired application, contributing to the acquisition of biological performance and function. The integration of instructive cues within tissue-engineered constructs will allow a better control with less manipulations of the whole system compared to the polymeric scaffolds.

Considering the above, the integration of Dex releasing feature within the PEOT/PBT electrospun template enables the development of a scaffold that can facilitate favorable cellular responses. Nevertheless, the release of drug/bioactive molecules from polymeric scaffold depends on various factors such as microstructure of scaffold, polymer composition, polymer hydrophilicity, drug loading capacity, degradation characteristic and polymer/drug interactions [63]. Thus, an ideal scaffold should have sustained release of the entrapped drug to direct the differentiation of cells. Moreover, it is also expected that the scaffold should be biodegradable and have high porosity to promote cell migration and diffusion of nutrients and waste products. Compared to a bulk polymer scaffold, electrospun scaffolds have a faster drug release characteristic due to a larger surface area [17]. Moreover, the interaction
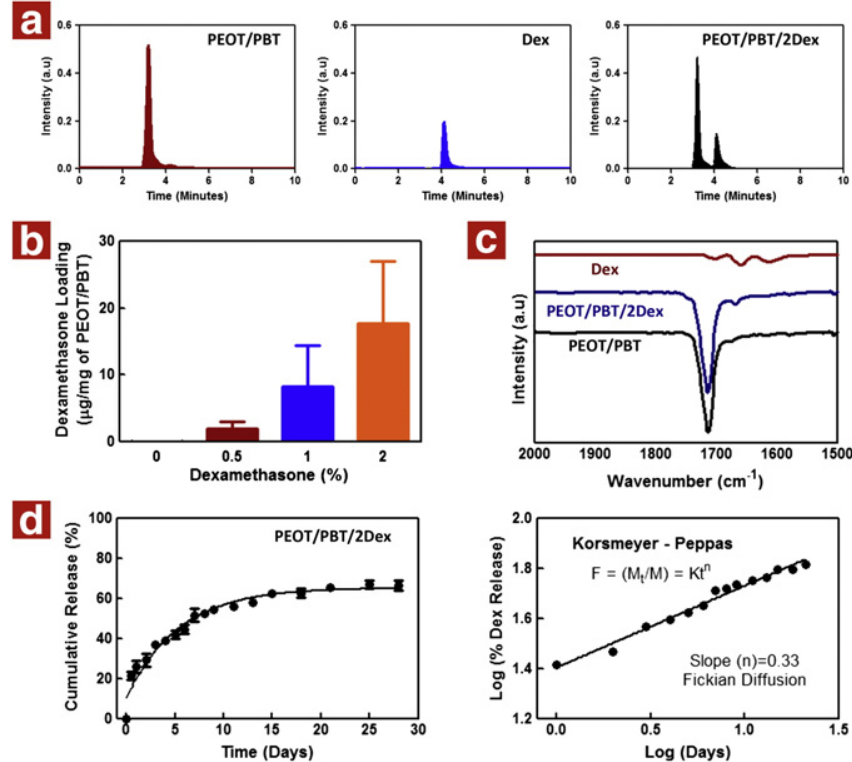

Fig. 3. Loading of Dex within PEOT/PBT fibers. (a) Identification of Dex and PEOT/PBT in HPLC. The Dex peak appears at $4.2 \mathrm{~min}$, whereas the PEOT/PBT peak appears around $3.3 \mathrm{~min}$ in HPLC. The Dex peak is quite far from the polymer peak and thus can be easily identified. PEOT/PBT/2Dex have a peak for both polymer and Dex indicating successful entrapment of drug within polymer scaffolds. (b) The loading of Dex within the polymeric scaffold was determined by dissolving the electrospun scaffold. The results indicate high entrapment efficiency of Dex within the fibrillar scaffolds. (c) The presence of Dex within the PEOT/PBT fibers was also confirmed by FTIR that shows a peak at $1660 \mathrm{~cm}^{-1}$. (d) The release of Dex from the electrospun scaffold was monitored in physiological conditions over the period of 28 days. The Dex released from the scaffolds is normalized to total Dex loading (empirically determined). The results indicate a sustained release of Dex from the fibrillar scaffold after the initial burst release. The release kinetic data correlate with the Korsmeyer-Peppas model of drug diffusion from the polymeric matrix. The results indicate Fickian diffusion of Dex from the fibrillar structure, as the diffusion coefficient $(n)$ is 0.33 . The data represents mean \pm standard deviation $(n=3)$. between polymer and water also plays an important role in drug loading and release profiles. Previous studies reported that compared to hydrophobic or hydrophilic polymers, amphiphilic polymers have higher drug loading and drug stability $[64,65]$. For example, hydrophilic drugs have limited solubility in hydrophobic polymers and vice versa. Whereas, amphiphilic polymers can strongly interact with many different types of drugs and proteins and can entrap them within their polymeric structure $[64,65]$.

Dex was entrapped within the fibrous scaffolds by blend ESP. The in vitro release of Dex from the electrospun scaffold containing 2\% Dex was monitored over a period of 28 days (Fig. 3d). Within the first $24 \mathrm{~h}$ a small burst release $(\sim 20 \%)$ of drug was observed. This initial burst may be due to localization of drug near the fiber surface. After the initial burst release, a sustained release of Dex was observed over the course of 28 days, compatible with the concentrations that are usually employed during standard osteogenic differentiation protocols $\left(10^{-8} \mathrm{M}\right)$. For example, each scaffold was approximately $2-3 \mu \mathrm{g}$ in weight and $500 \mu \mathrm{l}$ of medium was used for the in vitro study. The scaffold containing $2 \%$ Dex will have $\sim 46.8 \pm 6.3 \mathrm{ng}$ of Dex. According to the release profile and ignoring the burst release that corresponds to $20 \%$ of loaded Dex, $40 \%$ of Dex was released over the period of 28 days. For PEOT/PBT ( $2 \%$ Dex), the $40 \%$ of the entire payload corresponds to $18.72 \pm 2.52 \mathrm{ng}$ of Dex that was released over the period of 28 days. On other hand, if we subject the cells to a constant Dex conc. of $10^{-8} \mathrm{M}(1.962 \mathrm{ng} / 500 \mu \mathrm{l}$ of medium) for 28 days and change the medium every 3 days, then we will be using $~ 17.658 \mathrm{ng}$ of Dex. For PEOT/PBT scaffolds containing $0.5 \%$ and $1 \%$ Dex, the cumulative release of Dex is much lower compared to the concentrations that are usually employed during osteogenic differentiation.

The sustained release of Dex from PEOT/PBT scaffolds can be mainly attributed to drug diffusion or polymer degradation, or a combination of both. Earlier studies indicated that the PEOT/PBT copolymer starts to degrade by hydrolysis and complete in vivo degradation occurs over a period of 1 year [66]. Moreover, due to the amphiphilic nature of the polymer, solvent driven diffusion of the drug is expected. To determine the mechanism of Dex release from PEOT/PBT fibers, the release kinetic data was fitted to the Korsmeyer-Peppas model $\left(M_{t} / M_{\infty}=K t^{n}\right)$. Where " $M_{t} / M_{\infty}$ " is the fraction of Dex diffused at time " $t$ ", " $K$ " is the diffusion rate constant and " $n$ " is the diffusion exponent. The experimental data were plotted as log (cumulative \% drug release) versus log (time) as shown in Fig. 3d. The result indicates that the value of " $n$ " is 0.33 , implying Fickian diffusion of Dex from the electrospun PEOT/PBT fibrous matrix. Thus, we believe that the sustained release of Dex is driven by diffusion of the drug from the polymeric network.

\subsection{Effect of sustained release of Dex on hMSC adhesion and proliferation}

hMSCs are clinically relevant cells due to their multipotent nature and self-renewal ability $[67,68]$. hMSCs are in continuous and dynamic interaction with the surrounding extracellular matrix that dictates their behavior and functionality. Earlier studies have shown that cells elongate along the fiber axis and cellular morphology plays an important role in cellular behavior $[34,69]$. The interaction between hMSCs and electrospun scaffolds was evaluated by monitoring hMSC adhesion and proliferation on scaffolds. All the scaffolds allowed cellular adhesion and proliferation, as well as the organization of the cell body on the fibers. The cells were uniformly spread and elongated along the fiber axis as determined by microscopic analysis and staining of the cells cytoskeleton (Fig. 4a). The fiber morphology plays an important role in initial cell adhesion and spreading. It was observed that hMSCs readily attached and spread on fibers with a smaller fiber diameter (PEOT/PBT with Dex) compared to PEOT/PBT. All the scaffolds showed adsorption of protein when subjected to $10 \%$ FBS (Fig. $4 \mathrm{~b}$ ). Addition of Dex to PEOT/PBT resulted in smaller fiber diameter and larger surface area; this might be attributed to the enhanced protein adsorption on the electrospun scaffolds. 
a
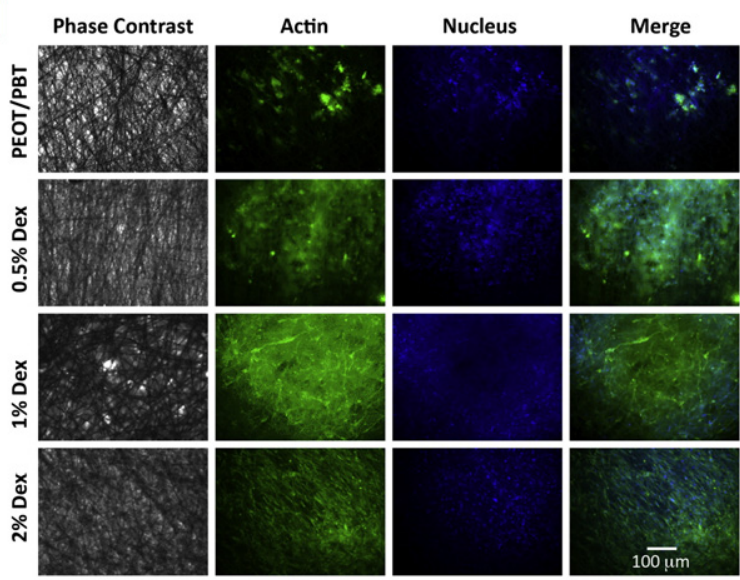

b
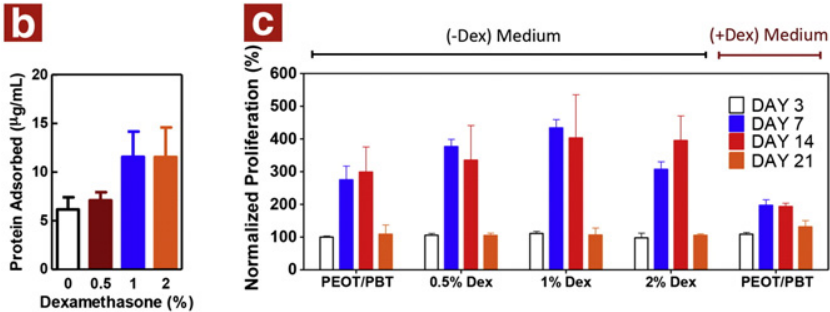

Fig. 4. Adhesion and proliferation of hMSCS on PEOT/PBT fibers. (a) hMSCs readily attach and spread on the fibrous structure. Cell bodies were stretched along the fiber axis. Compared to PEOT/PBT fibers, hMSCs were more spread on fiber containing Dex. (b) All the electrospun fibers adsorbed protein when submersed in $10 \%$ FBS. The fibers containing Dex (1 and 2\%) were observed to adsorb more protein compared to PEOT/PBT. This might be attributed to the enhanced surface area due to the small fiber diameter. (c) The proliferation of hMSCs was monitored over 21 days. The proliferation temporal profiles are in harmony with the ones corresponding to cells in the positive control subset (PEOT/PBT in (+ Dex) medium). The proliferation data for all the samples was normalized to proliferation of hMSCs on PEOT/PBT-day 3 ( - Dex) medium. The data represents mean \pm standard deviation $(n=3)$.

To investigate the effect of sustained release of Dex from PEOT/PBT scaffolds on metabolic activity, hMSCs were cultured in osteoconductive ( - Dex) and osteoinductive (+ Dex) medium. The osteoconductive ( - Dex) medium contain $\beta$-glycerophosphate and ascorbic acid. This medium formulation is able to support the functionality of osteoblast-like cells, mainly their ability to deposit matrix that will further be mineralized. The osteoinductive ( + Dex) medium contains $\beta$-glycerophosphate, ascorbic acid and dexamethasone. The addition of Dex $\left(10^{-8} \mathrm{M}\right)$ will provide the biochemical trigger towards the series of biochemical events that orchestrate the osteogenic differentiation. Within the scope of the study, the PEOT/PBT in ( - Dex) medium was used as negative control and PEOT/PBT in ( + Dex) medium was used as positive control.

During osteogenic differentiation, the metabolic activity of cells possesses a temporal component. During the first stage, the cells have an increased proliferation rate that is followed by a decrease, due to the switch of metabolism towards the osteogenic cellular commitment and maturation [48-50]. The metabolic activity of hMSCs cultured on electrospun PEOT/PBT scaffolds, monitored using Alamar Blue assay, is depicted in Fig. 4c. The metabolic activity of hMSCs cultured on the different experimental subsets showed a typical bell-shape pattern, consistent with the hypothesis mentioned above. A significant difference in the metabolic activity of hMSCs seeded on PEOT/PBT cultured in the absence and presence of Dex could be observed. The suppression of metabolic activity in PEOT/PBT (+ Dex) compared to PEOT/PBT (-Dex) is mainly attributed to the osteogenic differentiation of hMSCs. Due to the addition of Dex to the PEOT/PBT scaffolds, a change in metabolic activity on day 7 was observed. At lower Dex concentration
(PEOT/PBT/0.5Dex) a significant increase in metabolic activity was observed. It might be possible that topography (smaller fiber diameter) might be responsible for the enhanced metabolic activity. As the amount of Dex increased, the metabolic activity of hMSCs decreased (day 7). Taken together, these results highlight that PEOT/PBT electrospun scaffolds support hMSC adhesion, spreading and proliferation,-primary requirements to promote relevant biological behaviors in tissue engineering.

\subsection{Effect of sustained release of Dex on osteogenic differentiation of hMSCS}

The differentiation of hMSCs seeded on fibrous scaffold was investigated by monitoring ALP activity over the course of 28 days (Fig. 5a and b). ALP is a mid-stage checkpoint for the osteogenic differentiation, whose expression profile follows a temporal coordinate. An increase of ALP activity normalized to the number of cells, until reaching a "peak", is accompanied by the matrix production. A decrease in the ALP activity corresponds to the formation of mineral nucleation sites that consist of inorganic calcium.

For the scaffolds without Dex, in ( - Dex) medium, a residual ALP activity was observed, that was kept constant during the experimental time frame (Fig. 5b). However, with the increase of the Dex loading, and therefore, with the increase of the released drug, an increase in the ALP activity can be observed starting at day 7, until reaching a maximum value at day 14 . At this time point, significantly higher ALP activity of hMSCs was observed on fibrous scaffolds containing $1 \%$ and $2 \%$
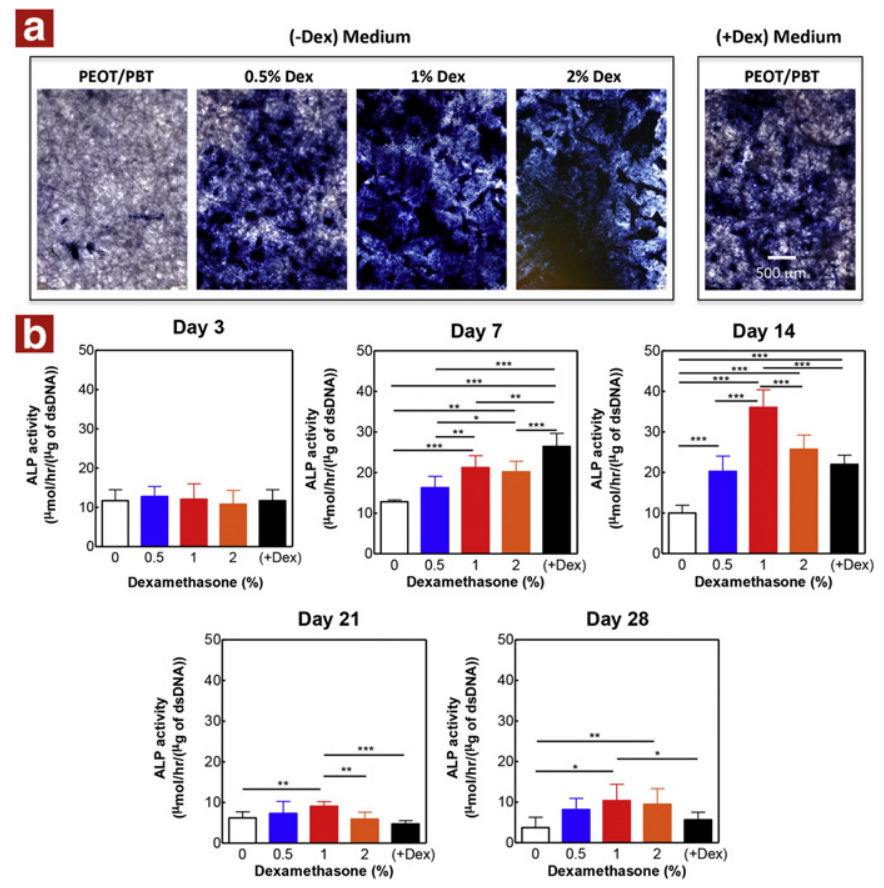

Fig. 5. Effect of Dex on ALP activity of hMSCs. hMSCs were cultured on the PEOT/PBT scaffold in osteoconductive medium ( - Dex) and osteoinductive ( + Dex) medium. Whereas PEOT/PBT scaffolds containing Dex were cultured only in osteoconductive ( - Dex) medium to evaluate the effect of Dex release on the ALP activity of hMSCs. (a) hMSCs stained for surface ALP-positive cells after 21 days. A uniform distribution of the ALP-positive cells on the scaffold can be observed, suggesting that the differentiation occurs in a homogeneous manner. (b) ALP activity of hMSCs seeded on electrospun scaffold was monitored over the period of 28 days. The ALP activity profile along time presents a bell shape pattern, compatible with osteogenic differentiation of hMSCs. Briefly, no significant effect of Dex was observed on day 3. On day 7 and 14 , scaffold-containing Dex show a significantly higher ALP activity compared to the negative control. On day 14 , the scaffold containing $1 \%$ Dex shows highest ALP activity; followed by a sharp decrease, until day 28 . This indicates that the sustained release of Dex from the polymeric scaffold triggers and sustains the osteogenic differentiation of stem cells. The bars represent mean \pm standard deviation $(n=3)$ (One-way ANOVA with Tukey post-hoc, ${ }^{*} p<0.05,{ }^{* *} p<0.01$ and ${ }^{* * *} p<0.001$ ). 
Dex, when compared with the positive control (PEOT/PBT in (+ Dex) medium). The scaffolds containing $0.5 \%$ and $2 \%$ Dex showed ALP activity similar to the positive control, indicating that the Dex release from the scaffolds can trigger and sustain the commitment of hMSCs towards osteogenic differentiation. This also indicates that the rate of Dex release from scaffolds has a similar or enhanced influence on up-regulation of ALP activity when hMSCs are subjected to a continuous and constant level of Dex.

To further evaluate the effect of Dex release on the differentiation of hMSC, the extent of the production of a mineralized matrix was evaluated, by the Alizarin Red staining [70]. The mineralized matrix consists of calcium deposits, and underlines the osteoblast-like cells functionality acquired by the differentiated hMSCs. This end-point is the hallmark of complete stabilization and maturation of the differentiated cells. Fig. 6 indicates that PEOT/PBT scaffolds without Dex (negative control) did not induce osteogenic differentiation of hMSCs, whereas PEOT/PBT scaffolds subjected to ( + Dex) (positive control) facilitate the formation of mineralized matrix. We further quantified the amount of the mineralized matrix by analyzing the area of the stained region (Fig. 6b). The results correlated well with the ALP activity of hMSCs and the scaffolds containing $1 \%$ Dex showed the highest area fraction of stained region compared to all other scaffolds, whereas scaffolds containing 0.5 and $2 \%$ Dex have similar mineralized area fractions similar to the positive control.
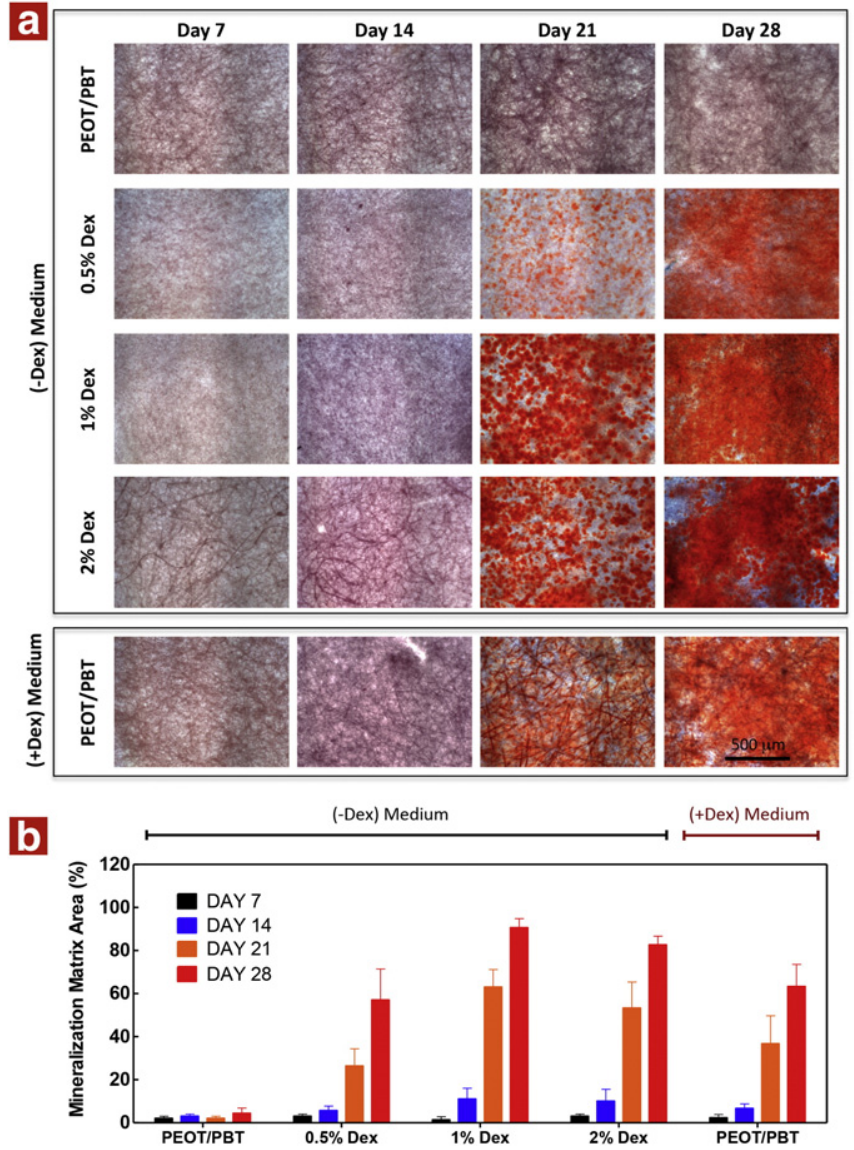

Fig. 6. Effect of Dex on production of the mineralized matrix. Alizarin Red was used to stain inorganic calcium deposition to identify production of the mineralized matrix by hMSCs. PEOT/PBT cultured in (-Dex) medium does not show any mineralized matrix indicating no spontaneous differentiation of seeded hMSCs. The PEOT/PBT in medium $(+$ Dex $)$ showed the formation of the mineralized matrix indicating production of the mineralized matrix by seeded hMSCs acting as positive control. The addition of Dex to polymeric scaffolds significantly enhances the production of the mineralized matrix. (b) The image quantification indicates that scaffolds containing $1 \%$ Dex showed the highest amount of mineralized matrix on day 21 and 28 compared to the positive control PEOT/PBT in ( + Dex $)$ medium. The data represents mean \pm standard deviation $(n=3)$.

\section{Conclusions}

We introduce electrospun scaffolds with a beaded structure as drug reservoirs for tissue engineering applications. Dexamethasone, as a model drug, was encapsulated within PEOT/PBT multi-block amphiphilic copolymer and the effect of drug entrapment was investigated on some of the physical, chemical and biological properties. The sustained release of Dex from the beaded structure was observed over the course of 21 days. The effect of the initial drug loads and the subsequent sustained release of Dex on human bone marrow stem cell differentiation were also investigated. The fibrous scaffolds containing Dex upregulate ALP activity and facilitate the formation of the mineralized matrix, without the addition of Dex in the culture medium. The electrospun scaffolds with a beaded fibrous structure can potentially be used to deliver bioactive agents for regenerative medicine within a controlled and continuous fashion.

\section{Acknowledgments}

AKG, SMM, LM and AK conceived the idea and designed the experiments. AKG and SMM fabricated electrospun scaffolds and performed the structural (SEM, FTIR), mechanical, and in vitro studies. AAK and AKG performed Dex release study. AKG and AP performed thermal analysis. AKG analyzed experimental data. AKG, SMM, LM and AK wrote the manuscript. ADL and CVB provided the polymers and corrected the manuscript. AKK, AP, MG and RLR revised the paper. All authors discussed the results and commented on the manuscript. Authors would like to thank Shilpaa Mukundan, Poornima Kulkarni and Dr. Arghya Paul for help with image analysis, drug release modeling and technical discussion respectively. AKG would like to thank Prof. Robert Langer for access to equipment and acknowledge financial support from MIT Portugal Program (MPP-09Call-Langer-47). SMM thanks the Portuguese Foundation for Science and Technology (FCT) for the personal grant SFRH/BD/42968/2008 (MIT-Portugal Program). This research was funded by the office of Naval Research Young National Investigator Award (AK), the Presidential Early Career Award for Scientists and Engineers (PECASE) (AK), the NIH (EB009196; DE019024; EB007249; HL099073; AR057837), the National Science Foundation CAREER award (DMR 0847287; AK), and the Dutch Technology Foundation (STW \#11135; LM, CVB, and AD).

\section{References}

[1] L.G. Griffith, G. Naughton, tissue engineering-current challenges and expanding opportunities, Science 295 (2002) 1009-1014.

[2] A. Khademhosseini, J.P. Vacanti, R. Langer, Progress in tissue engineering, Sci. Am. 300 (2009) 64-71.

[3] R. Langer, J.P. Vacanti, Tissue Engineering, Science 260 (1993) 920-926.

[4] N.A. Peppas, J.Z. Hilt, A. Khademhosseini, R. Langer, Hydrogels in biology and medicine: from molecular principles to bionanotechnology, Adv. Mater. 18 (2006) 1345-1360.

[5] B.V. Slaughter, S.S. Khurshid, O.Z. Fisher, A. Khademhosseini, N.A. Peppas, Hydrogels in regenerative medicine, Adv. Mater. 21 (2009) 3307-3329.

[6] W.J. Li, C.T. Laurencin, E.J. Caterson, R.S. Tuan, F.K. Ko, Electrospun nanofibrous structure: a novel scaffold for tissue engineering, J. Biomed. Mater. Res. 60 (2002) 613-621.

[7] H. Yoshimoto, Y. Shin, H. Terai, J. Vacanti, A biodegradable nanofiber scaffold by electrospinning and its potential for bone tissue engineering, Biomaterials 24 (2003) 2077-2082.

[8] J.M. Holzwarth, P.X. Ma, Biomimetic nanofibrous scaffolds for bone tissue engineering, Biomaterials 32 (2011) 9622-9629.

[9] Q.P. Pham, U. Sharma, A.G. Mikos, Electrospinning of polymeric nanofibers for tissue engineering applications: a review, Tissue Eng. 12 (2006) 1197-1211.

[10] H. Liu, H. Peng, Y. Wu, C. Zhang, Y. Cai, G. Xu, Q. Li, X. Chen, J. Ji, Y. Zhang, The promotion of bone regeneration by nanofibrous hydroxyapatite/chitosan scaffolds by effects on integrin-BMP/Smad signaling pathway in BMSCs, Biomaterials 34 (2013) 4404-4417.

[11] J. Xue, B. Feng, R. Zheng, Y. Lu, G. Zhou, W. Liu, Y. Cao, Y. Zhang, W.J. Zhang Engineering ear-shaped cartilage using electrospun fibrous membranes of gelatin/ polycaprolactone, Biomaterials 34 (2013) 2624-2631.

[12] Y. Xu, S. Dong, Q. Zhou, X. Mo, L. Song, T. Hou, J. Wu, S. Li, Y. Li, P. Li, The effect of mechanical stimulation on the maturation of TDSCs-poly(L-lactide-co-e-caprolactone)/ 
collagen scaffold constructs for tendon tissue engineering. Biomaterials 35 (2014) 2760-2772.

[13] G. Kim, J. Son, S. Park, W. Kim, Hybrid process for fabricating 3D hierarchical scaffolds combining rapid prototyping and electrospinning, Macromol. Rapid Commun. 29 (2008) 1577-1581.

[14] C. Li, C. Vepari, H.-J. Jin, H.J. Kim, D.L. Kaplan, Electrospun silk-BMP-2 scaffolds for bone tissue engineering, Biomaterials 27 (2006) 3115-3124.

[15] C.L. Casper, N. Yamaguchi, K.L. Kiick, J.F. Rabolt, Functionalizing electrospun fibers with biologically relevant macromolecules, Biomacromolecules 6 (2005) 1998-2007.

[16] K.Y. Lee, L. Jeong, Y.O. Kang, S.J. Lee, W.H. Park, Electrospinning of polysaccharides for regenerative medicine, Adv. Drug Deliv. Rev. 61 (2009) 1020-1032.

[17] T.J. Sill, H.A. von Recum, Electrospinning: applications in drug delivery and tissue engineering, Biomaterials 29 (2008) 1989-2006.

[18] A.K. Ekaputra, G.D. Prestwich, S.M. Cool, D.W. Hutmacher, Combining electrospun scaffolds with electrosprayed hydrogels leads to three-dimensional cellularization of hybrid constructs, Biomacromolecules 9 (2008) 2097-2103.

[19] H. woon Choi, J.K. Johnson, J. Nam, D.F. Farson, J. Lannutti, Structuring electrospun polycaprolactone nanofiber tissue scaffolds by femtosecond laser ablation, J. Laser. Appl. 19 (2007) 225-231.

[20] H.S. Yoo, T.G. Kim, T.G. Park, Surface-functionalized electrospun nanofibers for tissue engineering and drug delivery, Adv. Drug Deliv. Rev. 61 (2009) 1033-1042.

[21] P. Rujitanaroj, Y.C. Wang, J. Wang, S.Y. Chew, Nanofiber-mediated controlled release of siRNA complexes for long term gene-silencing applications, Biomaterials 32 (2011) 5915-5923.

[22] S.H. Lim, H.Q. Mao, Electrospun scaffolds for stem cell engineering, Adv. Drug Deliv. Rev. 61 (2009) 1084-1096

[23] J. Zeng, X. Xu, X. Chen, Q. Liang, X. Bian, L. Yang, X. Jing, Biodegradable electrospun fibers for drug delivery, J. Control. Release 92 (2003) 227-231.

[24] J. Kim, I.S. Kim, T.H. Cho, K.B. Lee, S.J. Hwang, G. Tae, I. Noh, S.H. Lee, Y. Park, K. Sun, Bone regeneration using hyaluronic acid-based hydrogel with bone morphogenic protein-2 and human mesenchymal stem cells, Biomaterials 28 (2007) 1830-1837.

[25] D.S.W. Benoit, A.R. Durney, K.S. Anseth, The effect of heparin-functionalized PEG hydrogels on three-dimensional human mesenchymal stem cell osteogenic differentiation, Biomaterials 28 (2007) 66-77.

[26] C.R. Nuttelman, M.C. Tripodi, K.S. Anseth, Dexamethasone-functionalized gels induce osteogenic differentiation of encapsulated hMSCs, J. Biomed. Mater. Res. A 76A (2006) 183-195.

[27] J.J. Yoon, J.H. Kim, T.G. Park, Dexamethasone-releasing biodegradable polymer scaffolds fabricated by a gas-foaming/salt-leaching method, Biomaterials 24 (2003) 2323-2329.

[28] A.K. Gaharwar, S.A. Dammu, J.M. Canter, C.-J. Wu, G. Schmidt, Highly extensible, tough, and elastomeric nanocomposite hydrogels from poly(ethylene glycol) and hydroxyapatite nanoparticles, Biomacromolecules 12 (2011) 1641-1650.

[29] L. Sun, S.T. Parker, D. Syoji, X. Wang, J.A. Lewis, D.L. Kaplan, Direct-write assembly of 3D silk/hydroxyapatite scaffolds for bone co-cultures, Adv. Healthc. Mater. 1 (2012) 729-735.

[30] A. Nandakumar, L. Yang, P. Habibovic, C. van Blitterswijk, Calcium phosphate coated electrospun fiber matrices as scaffolds for bone tissue engineering, Langmuir 26 (2009) 7380-7387.

[31] A.K. Gaharwar, P. Schexnailder, V. Kaul, O. Akkus, D. Zakharov, S. Seifert, G. Schmidt Highly extensible bio-nanocomposite films with direction-dependent properties, Adv. Funct. Mater. 20 (2010) 429-436.

[32] A.K. Gaharwar, P.J. Schexnailder, B.P. Kline, G. Schmidt, Assessment of using Laponite cross-linked poly(ethylene oxide) for controlled cell adhesion and mineralization, Acta Biomater. 7 (2011) 568-577.

[33] A.K. Gaharwar, V. Kishore, C. Rivera, W. Bullock, C.J. Wu, O. Akkus, G. Schmidt, Physically crosslinked nanocomposites from silicate-crosslinked PEO: mechanica properties and osteogenic differentiation of human mesenchymal stem cells, Macromol. Biosci. 12 (2012) 779-793.

[34] L. Moroni, R. Licht, J. de Boer, J.R. de Wijn, C.A. van Blitterswijk, Fiber diameter and texture of electrospun PEOT/PBT scaffolds influence human mesenchymal stem cell proliferation and morphology, and the release of incorporated compounds, Biomaterials 27 (2006) 4911-4922.

[35] W. Cui, Y. Zhou, J. Chang, Electrospun nanofibrous materials for tissue engineering and drug delivery, Sci. Technol. Adv. Mater. 11 (2010) 014108.

[36] P.J. Barnes, I.M. Adcock, Glucocorticoid resistance in inflammatory diseases, Lancet 373 (2009) 1905-1917

[37] M.F. Pittenger, A.M. Mackay, S.C. Beck, R.K. Jaiswal, R. Douglas, J.D. Mosca, M.A Moorman, D.W. Simonetti, S. Craig, D.R. Marshak, Multilineage potential of adult human mesenchymal stem cells, Science 284 (1999) 143-147.

[38] Y. Jiang, B.N. Jahagirdar, R.L. Reinhardt, R.E. Schwartz, C.D. Keene, X.R. OrtizGonzalez, M. Reyes, T. Lenvik, T. Lund, M. Blackstad, Pluripotency of mesenchymal stem cells derived from adult marrow, Nature 418 (2002) 41-49.

[39] D. Rickard, T. Sullivan, B. Shenker, P. Leboy, I. Kazhdan, Induction of rapid osteoblast differentiation in rat bone marrow stromal cell cultures by dexamethasone and BMP-2, Dev. Biol. 161 (1994) 218-228.

[40] Y. Mikami, M. Lee, S. Irie, M.J. Honda, Dexamethasone modulates osteogenesis and adipogenesis with regulation of osterix expression in rat calvaria,Äêderived cells, J. Cell. Physiol. 226 (2011) 739-748.

[41] R.M. Porter, W.R. Huckle, A.S. Goldstein, Effect of dexamethasone withdrawal on osteoblastic differentiation of bone marrow stromal cells, J. Cell. Biochem. 90 (2003) 13-22.

[42] H. Oshina, S. Sotome, T. Yoshii, I. Torigoe, Y. Sugata, H. Maehara, E. Marukawa, K Omura, K. Shinomiya, Effects of continuous dexamethasone treatment on differentiation capabilities of bone marrow-derived mesenchymal cells, Bone 41 (2007) 575-583.

[43] T. Hickey, D. Kreutzer, D. Burgess, F. Moussy, Dexamethasone/PLGA microspheres for continuous delivery of an anti-inflammatory drug for implantable medical devices, Biomaterials 23 (2002) 1649-1656.

[44] T. Murakami, K. Ajima, J. Miyawaki, M. Yudasaka, S. Iijima, K. Shiba, Drug-loaded carbon nanohorns: adsorption and release of dexamethasone in vitro, Mol. Pharm. 1 (2004) 399-405

[45] X. Luo, C. Matranga, S. Tan, N. Alba, X.T. Cui, Carbon nanotube nanoreservoir for controlled release of anti-inflammatory dexamethasone, Biomaterials 32 (2011) 6316-6323.

[46] J.M. Oliveira, R.A. Sousa, N. Kotobuki, M. Tadokoro, M. Hirose, J.F. Mano, R.L. Reis, $\mathrm{H}$. Ohgushi, The osteogenic differentiation of rat bone marrow stromal cells cultured with dexamethasone-loaded carboxymethylchitosan/poly(amidoamine) dendrimer nanoparticles, Biomaterials 30 (2009) 804-813.

[47] H. Zhang, A. Patel, A.K. Gaharwar, S.M. Mihaila, G.I. Iviglia, S. Mukundan, H. Bae, H. Yang, A. Khademhosseini, Hyperbranched polyester hydrogels with controlled drug release and cell adhesion properties, Biomacromolecules 14 (2013) 1299-1310.

[48] A. Martins, J.V. Araújo, R.L. Reis, N.M. Neves, Electrospun nanostructured scaffolds for tissue engineering applications, Nanomedicine 2 (2007) 929-942.

[49] L.T.H. Nguyen, S. Liao, C.K. Chan, S. Ramakrishna, Electrospun poly(L-lactic acid) nanofibres loaded with dexamethasone to induce osteogenic differentiation of human mesenchymal stem cells, J. Biomater. Sci. Polym. Ed. 23 (2012) 1771-1791.

[50] N.M. Vacanti, H. Cheng, P.S. Hill, J.D.T. Guerreiro, T.T. Dang, M. Ma, S. Watson, N.S. Hwang, R.S. Langer, D.G. Anderson, Localized delivery of dexamethasone from electrospun fibers reduces the foreign body response, Biomacromolecules 13 (2012) 3031-3038.

[51] A. Martins, A.R.C. Duarte, S. Faria, A.P. Marques, R.L. Reis, N.M. Neves, Osteogenic induction of hBMSCs by electrospun scaffolds with dexamethasone release functionality, Biomaterials 31 (2010) 5875-5885.

[52] N.Z. Mostafa, R. Fitzsimmons, P.W. Major, A. Adesida, N. Jomha, H. Jiang, H. Uludag, Osteogenic differentiation of human mesenchymal stem cells cultured with dexamethasone, vitamin D3, basic fibroblast growth factor, and bone morphogenetic protein-2, Connect. Tissue Res. 53 (2012) 117-131.

[53] A. Patel, A.K. Gaharwar, G. Iviglia, H. Zhang S. Mukundan, S.M. Mihaila, D. Demarchi, A. Khademhosseini, Highly elastomeric poly(glycerol sebacate)-co-poly(ethylene glycol) amphiphilic block copolymers, Biomaterials 34 (2013) 3970.

[54] A. Patel, K. Mequanint, The kinetics of dithiocarbamate-mediated polyurethaneblock-poly(methyl methacrylate) polymers, Polymer 50 (2009) 4464-4470.

[55] M.B. Claase, D.W. Grijpma, S.C. Mendes, J.D. de Bruijn, J. Feijen, Porous PEOT/PBT scaffolds for bone tissue engineering: preparation, characterization, and in vitro bone marrow cell culturing, J. Biomed. Mater. Res. A 64 (2002) 291-300.

[56] E.J.P. Jansen, J. Pieper, M.J.J. Gijbels, N.A. Guldemond, J. Riesle, L.W. Van Rhijn, S.K. Bulstra, R. Kuijer, PEOT/PBT based scaffolds with low mechanical properties improve cartilage repair tissue formation in osteochondral defects, J. Biomed. Mater. Res. A 89 (2009) 444-452.

[57] A.A. Deschamps, M.B. Claase, W.J. Sleijster, J.D. de Bruijn, D.W. Grijpma, J. Feijen, Design of segmented poly(ether ester) materials and structures for the tissue engineering of bone, J. Control. Release 78 (2002) 175-186.

[58] G. Beumer, C. Van Blitterswijk, D. Bakker, M. Ponec, Cell-seeding and in vitro biocompatibility evaluation of polymeric matrices of PEO/PBT copolymers and PLLA, Biomaterials 14 (1993) 598-604.

[59] G. Beumer, C. Van Blitterswijk, M. Ponec, Degradative behaviour of polymeric matrices in (sub) dermal and muscle tissue of the rat: a quantitative study, Biomaterials 15 (1994) 551-559.

[60] A. Radder, H. Leenders, C. Van Blitterswijk, Application of porous PEO/PBT copolymers for bone replacement, J. Biomed. Mater. Res. 30 (1998) 341-351.

[61] L. Moroni, R. Schotel, D. Hamann, J.R. de Wijn, C.A. van Blitterswijk, 3D fiber deposited electrospun integrated scaffolds enhance cartilage tissue formation, Adv, Funct. Mater. 18 (2007) 53-60.

[62] M. Biondi, F. Ungaro, F. Quaglia, P.A. Netti, Controlled drug delivery in tissue engineering, Adv. Drug Deliv. Rev. 60 (2008) 229-242.

[63] J.Venugopal, M.P. Prabhakaran, S. Low, A.T. Choon, Y.Zhang, G. Deepika, S. Ramakrishna, Nanotechnology for nanomedicine and delivery of drugs, Curr. Pharm. Des. 14 (2008) 2184-2200

[64] L.Y. Qiu, Y.H. Bae, Polymer architecture and drug delivery, Pharm. Res. 23 (2006) $1-30$.

[65] M.L. Adams, A. Lavasanifar, G.S. Kwon, Amphiphilic block copolymers for drug delivery, J. Pharm. Sci. 92 (2003) 1343-1355.

[66] A. Deschamps, A. Van Apeldoorn, H. Hayen, J. De Bruijn, U. Karst, D. Grijpma, J. Feijen, In vivo and in vitro degradation of poly(ether ester) block copolymers based on poly(ethylene glycol) and poly(butylene terephthalate), Biomaterials 25 (2004) 247-258.

[67] Y Jiang B.N. Jahagirdar, R.L. Reinhardt, R.E. Schwartz, C.D. Keene, X.R. Ortiz-Gonzalez, M. Reyes, T. Lenvik, T. Lund, M. Blackstad, J. Du, S. Aldrich, A. Lisberg, W.C. Low, D.A. Largaespada, C.M. Verfaillie, Pluripotency of mesenchymal stem cells derived from adult marrow, Nature 418 (2002) 41-49.

[68] A.J. Engler, S. Sen, H.L. Sweeney, D.E. Discher, Matrix elasticity directs stem cell lineage specification, Cell 126 (2006) 677-689.

[69] A.K. Gaharwar, P.J. Schexnailder, A. Dundigalla, J.D. White, C.R. Matos-Pérez, J.L. Cloud, S. Seifert, J.J. Wilker, G. Schmidt, Highly extensible bio-nanocomposite fibers, Macromol. Rapid Commun. 32 (2011) 50-57.

[70] C.A. Gregory, W. Grady Gunn, A. Peister, D.J. Prockop, An Alizarin red-based assay of mineralization by adherent cells in culture: comparison with cetylpyridinium chloride extraction, Anal. Biochem. 329 (2004) 77-84. 